\title{
CONHECIMENTOS E PRÁTICAS SOBRE A SISTEMATIZAÇÃO DA ASSISTÊNCIA DE ENFERMAGEM NA ESTRATÉGIA SAÚDE DA FAMÍLIA*
}

Gisele de Castro Varela1, Suzana Carneiro de Azevedo Fernandes²

\begin{abstract}
RESUMO: A Sistematização da Assistência de Enfermagem constitui instrumento científico que norteia e viabiliza o trabalho do enfermeiro sendo importante no âmbito da Estratégia Saúde da Família, implantada a fim de instituir novas práticas de saúde. Os objetivos desta pesquisa qualitativa de caráter exploratório foram identificar a compreensão dos enfermeiros da Estratégia acerca da Sistematização e compreender como sistematizam a assistência de enfermagem na Estratégia; foi desenvolvida em 2010 com nove enfermeiros de três Unidades Básicas de Saúde da Família no Município de Mossoró - Rio Grande do Norte. A análise das entrevistas resultou nas categorias Compreensão dos enfermeiros acerca da SAE e Desenvolvimento da SAE nas atividades profissionais realizadas na Saúde da Família. Observou-se que embora os enfermeiros compreendam a importância da Sistematização, em meio a vários obstáculos, não a desenvolvem. Deve-se lançar mão de meios que viabilizem a assistência científica, diferenciada, dinâmica e facilitadora.
\end{abstract}

DESCRITORES: Enfermagem; Processos de enfermagem; Saúde da família.

\section{KNOWLEDGE AND PRACTICES OF THE SYSTEMATIZATION OF NURSING CARE IN THE FAMILY HEALTH STRATEGY}

\begin{abstract}
The Systematization of Nursing Care is a scientific instrument which guides and viabilizes the nurse's work, and is important in the ambit of the Family Health Strategy, implanted so as to institute new health practices. The objectives of this qualitative research with an exploratory character were to identify the nurses' understanding of the Strategy on Systematization, and understand how they systematize the nursing care in the Strategy; it was undertaken in 2010 with nine nurses from three Family Health Primary care centers in the municipality of Mossoró in the state of Rio Grande do Norte. The analysis of the interviews resulted in the categories Nurses' understanding on the SNC and Development of the SNC in the professional activities carried out in Family Health. It was observed that although the nurses understand the importance of Systematization, due to various obstacles they do not develop it. Means must be used which viabilize scientific, differentiated, dynamic and facilitative care.

DESCRIPTORS: Nursing; Nursing processes; Family health.

\section{CONOCIMIENTOS Y PRÁCTICAS SOBRE LA SISTEMATIZACIÓN DE LA ASISTENCIA DE ENFERMERÍA EN LA ESTRATEGIA SALUD DE LA FAMILIA}

RESUMEN: La Sistematización de la Asistencia de Enfermería constituye instrumento científico que da la dirección y vuelve viable el trabajo del enfermero y es importante en el ámbito de la Estrategia Salud de la Familia, implantada a fin de instituir nuevas prácticas de salud. Los objetivos de esta investigación cualitativa de carácter exploratorio fueron identificar la comprensión de los enfermeros de la Estrategia acerca de la Sistematización y comprender cómo sistematizan la asistencia de enfermería en la Estrategia; fue desarrollada en 2010 con nueve enfermeros de tres Unidades Básicas de Salud de la Familia en el Municipio de Mossoró - Rio Grande do Norte. El análisis de las entrevistas resultó en las categorías Comprensión de los enfermeros acerca de la SAE y Desarrollo de la SAE en las actividades profesionales realizadas en la Salud de la Familia. Se ha observado que a pesar de los enfermeros comprenderen la importancia de la Sistematización, delante de varios obstáculos, no la desarrollan. Es necesario utilizarse de medios que vuelvan viables la asistencia científica, diferenciada, dinámica y facilitadora.

DESCRIPTORES: Enfermería; Procesos de enfermería; Salud de la familia.

* Artigo originado de monografia de conclusão apresentada ao Curso Graduação em Enfermagem da Universidade do Estado do Rio Grande do Norte- UERN, em 2011.

${ }^{1}$ Enfermeira do Hospital da Mulher de Fortaleza-CE. Especialista em Unidade de Terapia Intensiva. Professora do Curso Técnico em Enfermagem do Colégio Presidente Kennedy - Limoeiro do Norte - CE.

${ }^{2}$ Enfermeira. Doutora em Ciências Sociais. Diretora da Faculdade de Enfermagem da UERN. Tutora do Programa de Educação Tutorial em Enfermagem de Mossoró - PETEM UERN. 


\section{INTRODUÇÃO}

Com a construção do Sistema Único de Saúde (SUS) foi implantada a Saúde da Família como uma estratégia de reorientação do modelo assistencial, a fim de reestruturar as ações e instituir novas práticas de saúde. A Estratégia Saúde da Família (ESF), operacionalizada mediante a implantação de equipes multiprofissionais em unidades básicas de saúde, busca transpor a visão fragmentada do ser humano para uma compreensão integral na dimensão individual, familiar e coletiva ${ }^{(1)}$.

O enfermeiro, como um importante membro da equipe básica multidisciplinar e por ser um componente ativo no processo de consolidação da ESF como política integrativa e humanizadora da saúde, necessita de instrumentos que viabilizem a sua prática profissional e a execução dos objetivos propostos pela Estratégia. Para tanto a Sistematização da Assistência de Enfermagem (SAE) mostra-se uma ferramenta essencial para atender aos princípios do SUS e nortear a prática e os objetivos da ESF. A SAE constitui instrumento metodológico que propicia e orienta a assistência de enfermagem, assim como oferece condições necessárias para a organização do trabalho do enfermeiro ${ }^{(2)}$.

A SAE surgiu quando a Enfermagem começou a sistematizar as suas ações com o intuito de integrar o seu conhecimento teórico à prática assistencial e, assim, construir um corpo de conhecimento autônomo na busca de consolidar a profissão enquanto ciência ${ }^{(3)}$. Com o advir de um saber científico próprio, a Enfermagem prima pela indissociabilidade entre o pensar, o ser e o agir, o que tira as amarras que a subjugam, bem como qualifica a prática assistencial ${ }^{(4)}$. A SAE está legitimada pela resolução 358/2009 que regulamenta sua implementação em todas as instituições públicas ou privadas em que ocorra o cuidado profissional de enfermagem ${ }^{(5)}$.

Existem métodos utilizados na prática assistencial para sua sistematização, sendo um deles o Processo de Enfermagem(PE). Este é um método de tomada de decisão, de forma deliberada, que se apoia nos passos do método científico e tem por objetivo prestar um cuidado dinâmico, interativo e humanizado. É de suma importância a aplicação do PE diante das contribuições que este proporciona ao paciente e ao próprio trabalho da enfermagem ${ }^{(6)}$.

O PE constitui-se na aplicação prática de uma teoria de Enfermagem ${ }^{(7)}$. As teorias surgiram para direcionar as ações dos enfermeiros de maneira a torná-los responsáveis pela assistência prestada ao paciente ${ }^{(8)}$. De acordo com a resolução 358/2009 do Conselho Federal de Enfermagem (COFEN), o PE está organizado em cinco etapas: coleta de dados histórico, diagnóstico, planejamento, implementação e avaliação de enfermagem $^{(5)}$. É válido ressaltar que estas etapas são contínuas e uma interpretação errônea de uma delas pode comprometer as demais. Embora estejam divididas didaticamente, as fases do Processo não ocorrem de forma isolada, ao contrário, estão inter-relacionadas e ocorrem ao mesmo tempo ${ }^{(8)}$.

Além disso, o PE não deve ser visualizado como algo já pronto e acabado que deve ser apenas seguido, sem reflexão crítica. Caso seja visto desta maneira estará voltado a uma assistência reducionista e fragmentada.

Como o PE se constitui em um dos métodos de operacionalizar a SAE é essencial que o mesmo se concretize na realidade com vistas à transformação das práticas assistenciais nos serviços de saúde. Caso contrário, constituirá em mais uma alternativa frustrada, sendo visualizado somente como mais um processo normativo/ legal ou burocrático, perdendo a sua essência ${ }^{(9)}$.

No contexto do novo paradigma de atenção à saúde, a SAE deve estar intrinsecamente relacionada com a autonomia profissional do enfermeiro, a autonomia do usuário e as necessidades biológicas e sociais da população ${ }^{(10)}$. Diante desse panorama da atenção básica, é necessário que a Enfermagem em Saúde Coletiva reveja o seu processo de sistematização das práticas a fim de que sejam alcançados os objetivos propostos pela $\mathrm{ESF}^{(10)}$.

O enfermeiro na ESF deve sistematizar a assistência conceituando pessoa como sendo o indivíduo, a família e/ou a comunidade; conceituando o ambiente de maneira que englobe a comunidade em que essa pessoa vive; conceituando saúde de acordo com as diretrizes da ESF e conceituando o profissional enfermeiro como um agente de promoção da saúde ${ }^{(8)}$.

Dentre as atribuições do enfermeiro que atua na ESF pode-se citar a realização da assistência integral atuando na promoção e proteção da saúde, diagnóstico, tratamento, reabilitação da saúde dos indivíduos e famílias, bem como realização da consulta de enfermagem e solicitação de exames complementares ${ }^{(11)}$. De acordo com a resolução 159/1993 do COFEN, a consulta de enfermagem é uma atividade privativa do enfermeiro que tem por objetivo identificar situações de saúde/doença com o intuito de diagnosticar e implementar medidas que contribuam para a promoção, prevenção, proteção da saúde, recuperação e reabilitação do indivíduo, família e comunidade, assim como promover e fortalecer o vínculo entre o profissional e o usuário ${ }^{(12)}$.

Considerando que na consulta o PE pode ser aplicado, e sendo a SAE operacionalizada tanto por meio 
do PE quanto da consulta de enfermagem, torna-se evidente e fundamental que o enfermeiro sistematize a assistência na ESF. Entretanto, segundo a Resolução 358/2009 do COFEN $^{(5)}$, o PE quando realizado em instituições prestadoras de serviços ambulatoriais, domicílios, entre outros, corresponde a própria consulta de enfermagem. Vale ressaltar que processo e consulta de enfermagem não se constituem em sinônimos, mas em ferramentas que podem ser utilizadas para implementar a sistematização da assistência de enfermagem ${ }^{(13)}$.

Portanto, cada cenário utilizará o método ou a ferramenta que melhor se adeque à realidade de determinado serviço de saúde, baseado na teoria mais adequada a fim de nortear a prática da enfermagem ${ }^{(13)}$.

Nesse sentido, torna-se de suma importância que o enfermeiro se aproprie da SAE na ESF, utilizando o processo ou a consulta de enfermagem ou, ainda, de ambos, de modo compartilhado com o usuário, estimulando o diálogo, o espaço de fala e escuta, fortalecendo o vínculo entre profissional/usuário bem como uma assistência acolhedora e humanizada.

Diante disso, esta pesquisa teve como objetivos identificar a compreensão dos enfermeiros da Saúde da Família acerca da Sistematização da Assistência de Enfermagem, bem como compreender como os enfermeiros sistematizam a assistência de enfermagem na Saúde da Família.

\section{MÉTODO}

Trata-se de uma pesquisa qualitativa do tipo exploratória. O estudo qualitativo possibilita abranger a totalidade do problema investigado em suas múltiplas dimensões. O estudo exploratório busca aperfeiçoar ideias e proporcionar novas discussões acerca do tema em questão ${ }^{(14)}$.

Para assegurar os aspectos éticos que envolvem as pesquisas com seres humanos, este trabalho foi submetido ao Comitê de Ética em Pesquisa da Universidade do Estado do Rio Grande do Norte, sendo aprovado segundo parecer n. $042 / 2010$, de 17/09/2010, CAAE n. 0040.0.428.000-10. O Termo de Consentimento Livre e Esclarecido, também aprovado no referido CEP, foi lido e explicado aos participantes antes do início das entrevistas, e assinado por todos.

Em seguida, deu-se início a coleta dos dados. Os critérios de seleção das USF foram: ser campo de estágio da Faculdade de Enfermagem da UERN e alocados em bairros diferentes. Após identificação das USF do Município de Mossoró, por campo de estágio e por bairro, selecionou-se, através de sorteio, três unidades de saúde para integrar a amostra do estudo.
Como critério de inclusão para seleção dos participantes da pesquisa elencou-se ser enfermeiro que atua na ESF e como critério de exclusão foi elencado enfermeiro afastado em gozo de licença especial, férias ou atestado médico. Como cada equipe possui três enfermeiros na sua composição, e todos os enfermeiros aceitaram participar do estudo, foram entrevistados 9 enfermeiros das ESF das unidades investigadas.

Os dados foram coletados a partir de entrevista semiestruturada, realizada de setembro a dezembro de 2010, por contato direto com os enfermeiros, mediante um agendamento prévio. Nessas foram abordadas questões abertas sobre a compreensão dos enfermeiros acerca da SAE, assim como o desenvolvimento da SAE nas atividades profissionais realizadas na Unidade. As entrevistas foram gravadas, sequencialmente foram transcritas, mantendo-se o sigilo, a privacidade e o anonimato dos participantes.

Para que o anonimato dos enfermeiros entrevistados fosse assegurado, os seus nomes foram substituídos e identificados por nomes de teóricas de enfermagem, por compreender que a construção das teorias constitui passo fundamental em direção à compreensão da Enfermagem como práxis social, transformadora do homem e da sociedade e um alicerce para a $\mathrm{SAE}^{(8)}$.

Os dados obtidos foram analisados por meio de categorias; estas se constituem em palavras expressivas em torno das quais o conteúdo de uma fala é organizado $^{(14)}$. Portanto, os dados foram agrupados segundo suas semelhanças e características comuns, produzindo duas categorias: Compreensão dos enfermeiros acerca da SAE e Desenvolvimento da SAE nas atividades profissionais realizadas na Saúde da Família. A construção de cada categoria foi subsidiada por palavras-chave extraídas de cada entrevista.

\section{RESULTADOS}

\section{Compreensão dos enfermeiros acerca da SAE}

As enfermeiras das USF, ao serem indagadas sobre a compreensão a respeito da SAE, demonstraram que possuem conhecimento do assunto, conforme relatos: É um método de planejar a assistência de enfermagem [...] para o indivíduo de acordo com o que ele apresenta. Fazemos um histórico, diagnóstico e traçamos metas para serem alcançadas para melhorar o estado do paciente. (Dorothea Orem)

Eu tenho a compreensão de que é uma forma de traba- 
Ihar na enfermagem, onde a gente organiza as nossas atividades e organiza também o nosso atendimento ao cliente. E assim, a compreensão é que é um pouco de teoria e de prática. (Martha Rogers)

Compreendo a SAE como a principal forma de organização do trabalho do enfermeiro. (Imogene King)

Eu compreendo como algo inerente ao processo de trabalho do enfermeiro, não tem como você não utilizar a SAE. É como um instrumento que serve para você prestar uma melhor assistência ao usuário [...] Então, você precisa da SAE para isso, é onde você vai canalizar melhor as ações [...] e fazer também com que a enfermagem como um todo, trabalhe direcionada pela SAE. E ai vai ter uma assistência melhor a esse usuário ou paciente de uma forma que cada um não trabalhe da sua maneira, mas sim de uma única maneira e assim, para se ter uma assistência de qualidade. (Wanda Horta)

A SAE é uma das ferramentas que a enfermagem tem como maior poder de autonomia de ver o processo do cuidar todo organizado. Os cuidados, os planos de cuidado que a gente vai desenvolver com aquele usuário, como ele respondeu e como ele está hoje [...]. A gente vai ver aquele usuário de forma individual e vai dar toda assistência a ele. Frente ao plano de cuidados que eu vou desenvolver intervenções/ações e resultados que eu vou colher frente a esse plano de intervenções. A SAE, na minha compreensão, é essa forma de intervir, de cuidar sistematicamente do usuário para poder ter uma melhor qualidade na assistência, e também melhorar a qualidade de vida desse usuário frente ao plano de cuidados que vai ser desenvolvido. (Madeleine Leininger)

\section{Desenvolvimento da SAE nas atividades profissio- nais realizadas na Saúde da Família}

As enfermeiras, ao serem questionadas sobre suas atividades profissionais na ESF, relataram a maneira como desenvolvem essas atividades e como estas são sistematizadas. Destacaram-se os discursos a seguir:

Dando assistência às gestantes e acompanhando mensalmente o decorrer da gestação com o sis-prenatal, o seu puerpério e o desenvolvimento de seu bebê através do $C$ e D, acompanhamento de idosos através do Programa de Hiperdia e do grupo de idosos existente nesta unidade de saúde, além dos programas de hanseníase e tuberculose
[...]. Há uma sistematização, pois, aos pacientes impossibilitados de vir à unidade de saúde, é feito um acompanhamento na residencia do paciente, independente de ser idoso, jovem, criança ou gestante [...]. (Myra Levine)

A sistematização na verdade ocorre assim: nós temos alguns programas que o Ministério da Saúde organiza, eles já vêm organizados para a gente trabalhar [...]. Então, a gente tem a divisão de horários, de cronograma de atendimentos para poder melhor se adequar a essas realidades. No caso, a ESF é diferente do hospital porque lá você trabalha com a sistematização de uma forma diferenciada, porque tem prescrição de enfermagem. Lá tem evolução de enfermagem e toda a sistematização através das anotações de enfermagem. (Martha Rogers)

As atividades são planejadas de acordo com os Programas de Atenção à Saúde do Ministério da Saúde e a realidade da população adscrita. (Hildegard Peplau)

Procuro sistematizar minha assistência baseada nas normas do Ministério da Saúde por meio dos manuais disponiveis nos programas. (Imogene King)

Sim, a gente tem tentado sempre fazer a Sistematização da Assistência de Enfermagem [...] porque assim, eu vou fazer C e D, eu sigo isso. Eu vou ver o que é importante para aquela criança, como aquela criança está inserida, o que ela está apresentando, e ai eu vou ver a questão de prevenção e tratamento também. Tudo isso vai para o prontuário, a gente não tem um pronto para isso, o que nós estamos querendo até implementar [...]. Então, assim, cada atividade realizada, seja um atendimento de pré-natal, seja um curativo, $C$ e D, a gente sempre tenta fazer uma Sistematização da Assistência de Enfermagem para que se tenha um tratamento contínuo e todo mundo fale a mesma coisa, que o usuário tenha uma assistência de qualidade e com os resultados que a gente espera que tenha. (Wanda Horta)

[...] sistematizo a assistência traçando metas focalizando o coletivo e, durante as consultas individuais, planejo a assistência individualizada. (Dorothea Orem)

\section{DISCUSSÃO}

Diante da primeira categoria, percebeu-se que na compreensão da enfermeira Orem a SAE é planejada de acordo com as necessidades do indivíduo. Desse modo, a partir do momento que o enfermeiro planeja a 
sua intervenção, conforme os problemas identificados, ele analisa quais precisam de atendimento imediato e aqueles cuja intervenção pode ser a médio ou longo prazo, o que dinamiza a assistência ${ }^{(8)}$.

Na compreensão da enfermeira Martha Rogers, visualizou-se que a SAE constitui-se em um processo de teoria/prática. Sabe-se que para a SAE ser operacionalizada é preciso que o enfermeiro tenha conhecimento das Teorias de Enfermagem, assim como se aproprie de uma filosofia de cuidado comprometida com a melhoria da assistência ${ }^{(15)}$. Esses aspectos correspondem à parte teórica, que é tão fundamental quanto à prática, pois as Teorias de Enfermagem funcionam como um alicerce para o processo de implementação da $\mathrm{SAE}^{(8)}$.

As Teorias podem ser consideradas como o alicerce epistemológico essencial à prática da enfermagem, uma vez que direcionam a assistência, assim como propiciam uma descrição e explicação dos aspectos da realidade, o que é basilar para a indissociabilidade da tríade teoria, prática e pesquisa ${ }^{(3)}$. Por outro lado, não se pode negar que a habilidade, a experiência e um arcabouço estrutural, recursos humanos e materiais (parte prática) também são essenciais para a efetivação da SAE.

Percebeu-se a necessidade de implantação da SAE quando a enfermeira Wanda Horta mencionou que com a SAE a enfermagem irá trabalhar de uma única maneira. Nesse sentido, entende-se que essa universalização da prática da enfermagem é relevante para o reconhecimento e valorização da profissão.

Um dos motivos que dificulta o reconhecimento da Enfermagem como ciência é a falta de uma linguagem compartilhada entre todos os enfermeiros e que estabeleça a definição e a descrição da prática desse profissional. Sem essa linguagem unificada não é possível visualizar a contribuição do trabalho do enfermeiro e de toda a sua equipe ${ }^{(16)}$. Assim, reafirma-se que é fundamental o profissional de enfermagem rever o seu processo de trabalho, repensando a sua maneira de agir em saúde. Afinal, é imprescindível primar por uma assistência sistematizada que visa uma abordagem organizada, resolutiva, integral.

Com relação à segunda categoria de análise, a partir dos depoimentos das enfermeiras, visualizou-se que a maneira como os enfermeiros sistematizam a assistência de enfermagem foi confundida com a descrição das atividades profissionais na ESF. Observou-se que o processo de sistematização da assistência se faz presente quando essas profissionais desenvolvem os programas preconizados pelo Ministério da Saúde.

Dessa maneira, visualizou-se que a SAE ainda se encontra restrita nos serviços de saúde, é como se bastasse colocar em prática os programas organizados pelo Ministério da Saúde. Assim, o agir em saúde dos enfermeiros contempla de maneira incipiente a SAE.

As enfermeiras, exceto Martha Rogers, não falaram acerca das fases que compõe o PE, um dos métodos que operacionaliza a SAE. Este fato pode estar relacionado à falta de conhecimento acerca do Processo, o que dificulta o entendimento da própria prática da enfermagem. Somente a enfermeira Martha Rogers mencionou algumas fases que o compõem, porém vinculou-as ao ambiente hospitalar, como se somente fosse realizado no hospital.

Talvez a maior parte da enfermagem não valorize a SAE na ESF porque não possui uma compreensão da dimensão individual na saúde coletiva, ficando a sistematização limitada ao espaço hospitalar, no qual o aspecto individual é supervalorizado em detrimento dos demais. É imprescindível romper com essa visão reducionista entendendo estes espaços como únicos e indissociáveis do cuidar em saúde.

É devido a essa visão limitada que, na maioria das vezes, as consultas, os atendimentos realizados não são valorizados pelos profissionais. Os mesmos cumprem somente uma função burocrática, na qual é importante gerar estatísticas, o que resulta na ausência de diagnósticos das necessidades de saúde, de planejamento sistematizado e de avaliação das ações realizadas tanto por parte dos profissionais quanto da comunidade ${ }^{(17)}$.

Isso tem culminado no desenvolvimento de uma atenção reducionista e fragmentada, o que vai de encontro às propostas da ESF. Esta vem desenvolvendo uma prática clínica pobre que realiza ações curativas superficiais e limitadas ${ }^{(18)}$. Prevalece ainda no âmbito da discussão da ESF uma dicotomia entre saúde coletiva e clínica, como se esta última não merecesse as propostas assistenciais estabelecidas no âmbito da saúde coletiva ${ }^{(18)}$.

O relato da enfermeira Wanda Horta evidenciou a necessidade de documentar a SAE, ao mencionar que todas as ações desenvolvidas devem ir para um prontuário; que, embora não exista um que contemple todas as anotações necessárias, mas que os profissionais estão tentando construir. $\mathrm{O}$ registro da assistência de enfermagem traz várias contribuições para a profissão, sendo, portanto, essencial colocar em prática essa documentação.

O descaso com o registro sistemático dos elementos que compõe a SAE pode resultar tanto em ausência de visibilidade e reconhecimento profissional, como em algo que pode ser ainda mais sério, ausência ou dificuldade de avaliação da prática da enfermagem ${ }^{(2)}$. 
Diante da fala da enfermeira Dorothea Orem compreendeu-se que o indivíduo tem que ser visualizado dentro de um contexto, por exemplo, familiar, e que, por sua vez, está inserido em um contexto maior, a comunidade, e assim sucessivamente. Considerando que o enfoque no coletivo é um dos objetivos da ESF, é preciso planejar intervenções que interfiram no âmbito do coletivo.

Considerando que a SAE busca contemplar a individualidade e particularidades do ser humano, é preciso também que se proponham ações em nível individual, com o intuito de alcançar uma melhoria no estado de saúde. Portanto, é fundamental que sejam contempladas ações tanto, em nível individual quanto coletivo.

\section{CONCLUSÃO}

O presente estudo permitiu visualizar que os enfermeiros entrevistados, de maneira geral, conhecem a SAE. Alguns, entretanto, têm uma visão ainda limitada e restrita; outros compreendem a SAE de maneira ampla e a consideram um instrumento de organização que facilita e dinamiza a assistência.

É preciso que os profissionais avancem na perspectiva de que sistematizar não é somente efetivar os programas que são estabelecidos pelo Ministério da Saúde. É relevante compreender que a SAE perpassa por outras dimensões, caso contrário, a Enfermagem estará embasando o seu cuidado em um saber limitado e fragmentado.

A SAE na ESF caminha a passos lentos, já que alguns profissionais ainda visualizam o cuidado a partir de dois espaços, a saúde coletiva e a assistência hospitalar. $\mathrm{Na}$ compreensão destes a SAE está relacionada ao âmbito hospitalar. É necessário que novas pesquisas sejam realizadas em relação a SAE na ESF, com a finalidade de promover o desenvolvimento e aprimoramento das práticas de enfermagem neste cenário. Afinal, primar por uma assistência comprometida com os princípios do SUS é o objetivo principal da ESF, assim como dos profissionais de saúde. Da mesma maneira, é imprescindível dispor de uma metodologia de cuidado que busca a qualidade do serviço, reconhecimento e autonomia profissional.

\section{REFERÊNCIAS}

1. Costa GD, Cotta RMM, Ferreira MLSM, Reis JR, Franceschini SCC. Saúde da família: desafios no processo de reorientação do modelo assistencial. Rev Bras Enferm. 2009;62(1):113-8.
2. Garcia TR, Nóbrega MML. Sistematização da Assistência de Enfermagem: reflexões sobre o processo. In: Anais do $52^{\circ}$ Congresso Brasileiro de Enfermagem. Recife/Olinda, Brasil. Recife/Olinda: ABEn/PE; 2000.

3. Schaurich D, Grossetti MGO. Produção do conhecimento sobre teorias de enfermagem: análise de periódicos da área, 1998-2007. Esc. Anna Nery. 2010;14(1):182-88.

4. Kletemberg DF, Mantovani MF, Lacerda MR. Entre a teoria e as práticas do cuidar: que caminho trilhar? Cogitare enferm. 2004;9(1):94-9.

5. Conselho Federal de Enfermagem. Resolução n. 358, de 15 de outubro de 2009. Dispõe sobre a Sistematização da Assistência de Enfermagem e a implementação do processo de enfermagem em ambientes, públicos ou privados, em que ocorre o cuidado profissional de enfermagem, e dá outras providências. Rio de Janeiro: COFEN; 2009.

6. Andrade JS, Vieira MJ. Prática assistencial de enfermagem: problemas, perspectivas e necessidade de sistematização. Rev Bras Enferm. 2005;58(3):261-5.

7. Hermida PMV. Desvelando a implementação da sistematização da assistência de enfermagem. Rev Bras Enferm. 2004;57(6):733-7.

8. Tannure MC, Gonçalves AMP. SAE, Sistematização da Assistência de Enfermagem: guia prático. Rio de Janeiro: Guanabara Koogan;2008.

9. Cianciarullo TI, Gualda DMR, Melleiro MM, Anabuki MH. Sistema de Assistência de Enfermagem: evolução e tendências. São Paulo: Ícone; 2001.

10. Barros DG, Chiesa AM. Autonomia e necessidades de saúde na Sistematização da Assistência de Enfermagem no olhar da saúde coletiva. Rev Esc Enferm. USP. 2007;41(n. esp):793-8.

11. Ministério da Saúde (BR). Secretaria de Atenção à Saúde. Departamento de Atenção Básica. Política Nacional de Atenção Básica. [Internet] 2006 [acesso em 18 dez 2010]. Disponível: http://bvsms.saude.gov.br/bvs/publicacoes/ politica_nacional_atencao_basica_2006.pdf

12. Conselho Federal de Enfermagem. Resolução n. 159, de 19 de abril de 1993. Dispõe sobre a Consulta de Enfermagem. Rio de Janeiro: COFEN; 1993.

13. Fuly PSC, Leite JL, Lima SBS. Correntes de pensamento nacionais sobre sistematização da assistência de enfermagem. Rev Bras Enferm. 2008;61(6):883-7. 
14. Minayo MCS. O desafio do conhecimento: pesquisa qualitativa em saúde. 10ª ed. São Paulo: Hucitec; 2007.

15. Backes DS, Schwartz E. Implementação da Sistematização da Assistência de Enfermagem: desafios e conquistas do ponto de vista gerencial. Cienc. cuid. saude. [Internet] 2005;4(2):182-8 [acesso em $18 \mathrm{dez}$ 2010]. Disponível: http://eduem.uem.br/ojs/index.php/ CiencCuidSaude/article/download/5247/3374

16. Albuquerque LM, Cubas MR, organizadoras. Cipescando em Curitiba: construção e implementação da nomenclatura de diagnósticos e intervenções de enfermagem na rede básica de saúde. ABEN. [Internet] 2005 [acesso em 18 dez 2010]. Disponível: ww.aben-pr. org.br/Arquivos/CIPESC.pdf

17. Shimizu HE, Rosales C. As práticas desenvolvidas no programa saúde da família contribuem para transformar o modelo de atenção a saúde? Rev Bras Enferm. 2009;62(14):424-9.

18. Franco TB, Merhy EE. Programa de Saúde da Família (PSF): contradições de um programa destinado à mudança do modelo tecnoassistencial. In: Merhy EE, Magalhães Junior HM, Franco TB, Bueno WS. O trabalho em saúde: olhando e experienciando o SUS no cotidiano. $4^{\mathrm{a}}$ ed. São Paulo: Hucitec; 2007. p.55-124. 